\title{
The CODE MGEX orbit and clock solution.
}

L. Prange, R. Dach , S. Lutz, S. Schaer, A. Jäggi

Astronomical Institute, University of Bern, Switzerland

IAG Scientific Assembly 2013, 1 - 6 September 2013,

Potsdam, Germany 


\section{Contents}

- MGEX network and data

- CODE MGEX orbit solution

- CODE MGEX clock solution

- Precise point positioning (PPP)

- Summary 


\section{MGEX network and data}

- Data sources: IGS: CDDIS, BKG, IGN (MGEX plus RINEX3 directories); EUREF: BKG (RINEX3 directory)

- Number of daily files/stations: about 100 files per day in mid 2013

- RINEX versions: $2.11,2.12,3.00,3.01,3.02$

- For some stations RINEX2 and RINEX3 are available

- Established IGS stations and new stations

- Public access to MGEX monitoring results via FTP: $=>\mathrm{ftp}: / / \mathrm{ftp}$.unibe.ch/aiub/mgex/ 


\section{MGEX network and data}

\section{Satellite systems being monitored (RINEX3 files):}

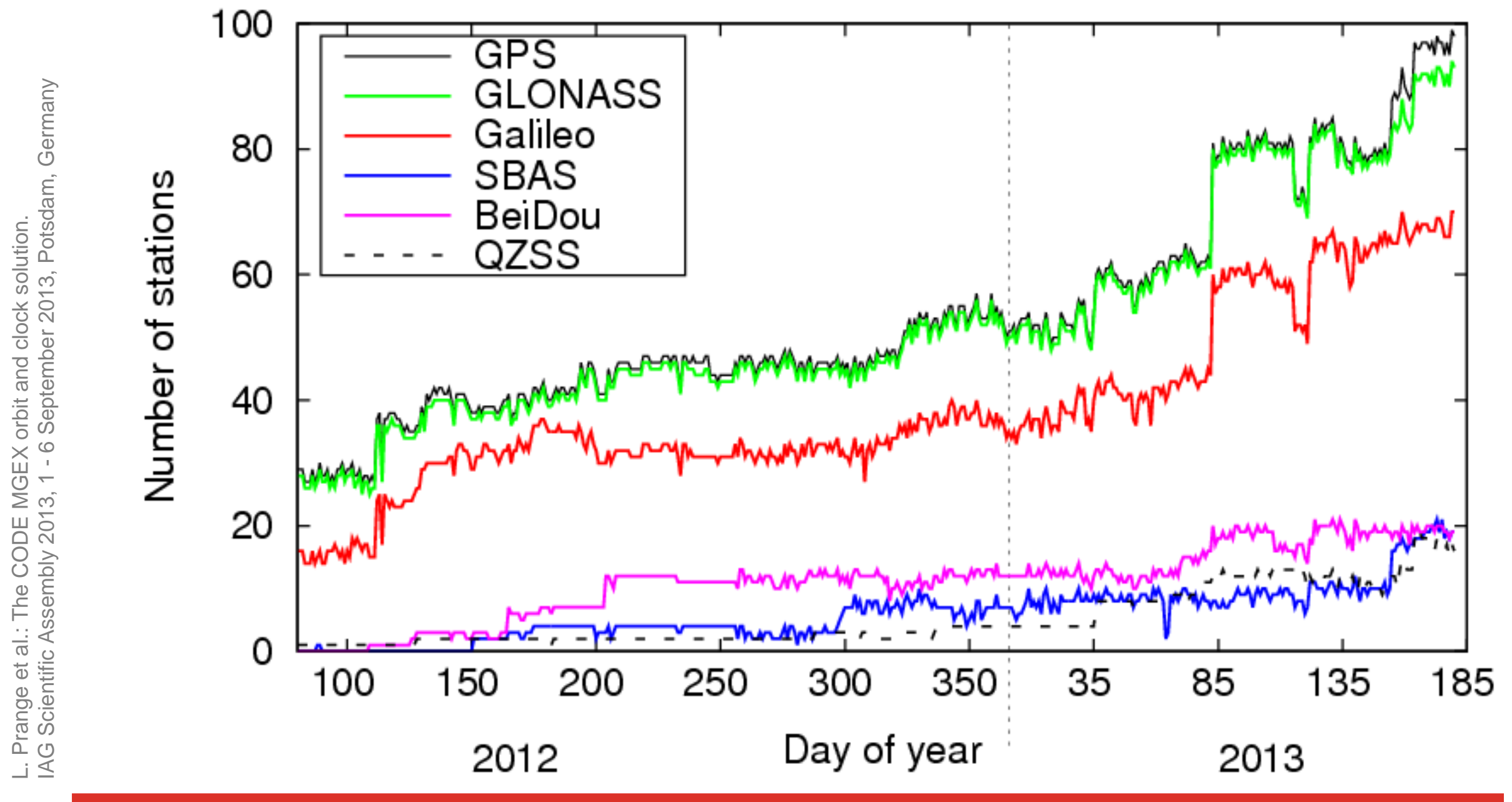




\section{CODE MGEX orbit solution: overview}

GNSS considered: GPS + GLONASS + Galileo (up to 60 satellites)

Processing mode: post-processing

Timespan covered: $\quad$ GPS-weeks 1689-1746 (DOY 12/146-13/180)

Number of stations: 150 (GPS + GLONASS),

30 - 40 (Galileo)

Processing scheme: double-difference network processing (observable: phase double differences)

Signal frequencies: L1+ L2 (GPS + GLONASS),

$\mathrm{E} 1$ (L1) + E5a (L5) (Galileo)

Orbit characteristic: 3-day long arcs

Reference frame: $\quad$ IGS08 (until week 1708); IGb08 (since week 1709)

IERS conventions: IERS2003 (until 1705); IERS2010 (since 1706)

Product list:

daily orbits (SP3) and ERPs

Distribution:

Designator:

ftp://cddis.gsfc.nasa.gov/gnss/products/mgex/ comwwwwd.???.Z 


\section{CODE MGEX orbit solution: station selection}

Number and distribution of tracking stations contributing to the CODE MGEX orbit solution (late 2012)

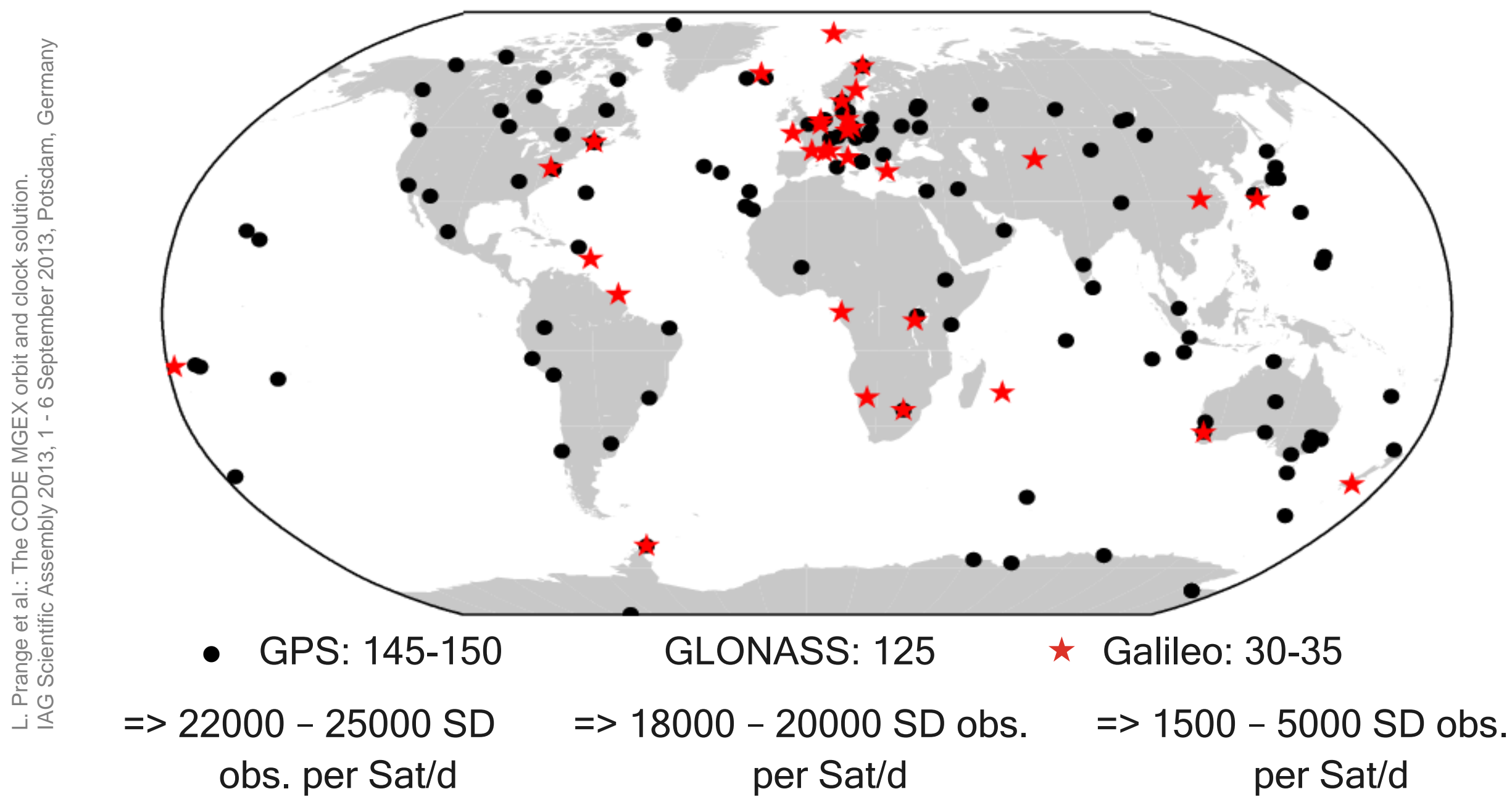




\section{CODE MGEX orbit solution: station selection}

Number and distribution of tracking stations contributing to the CODE MGEX orbit solution (mid 2013)

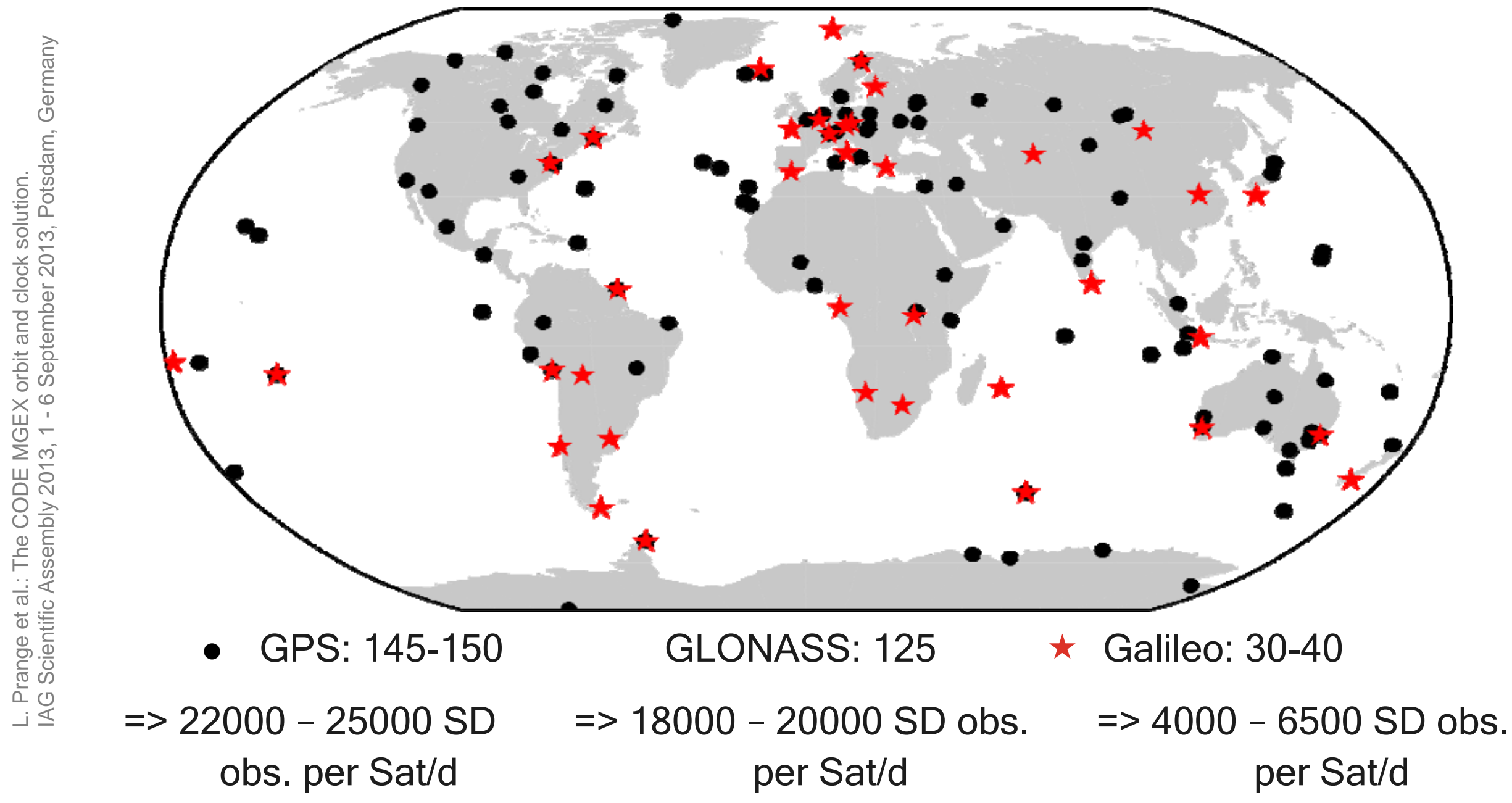




\section{Orbit validation: overlaps}

\section{Arclength: 1 day}

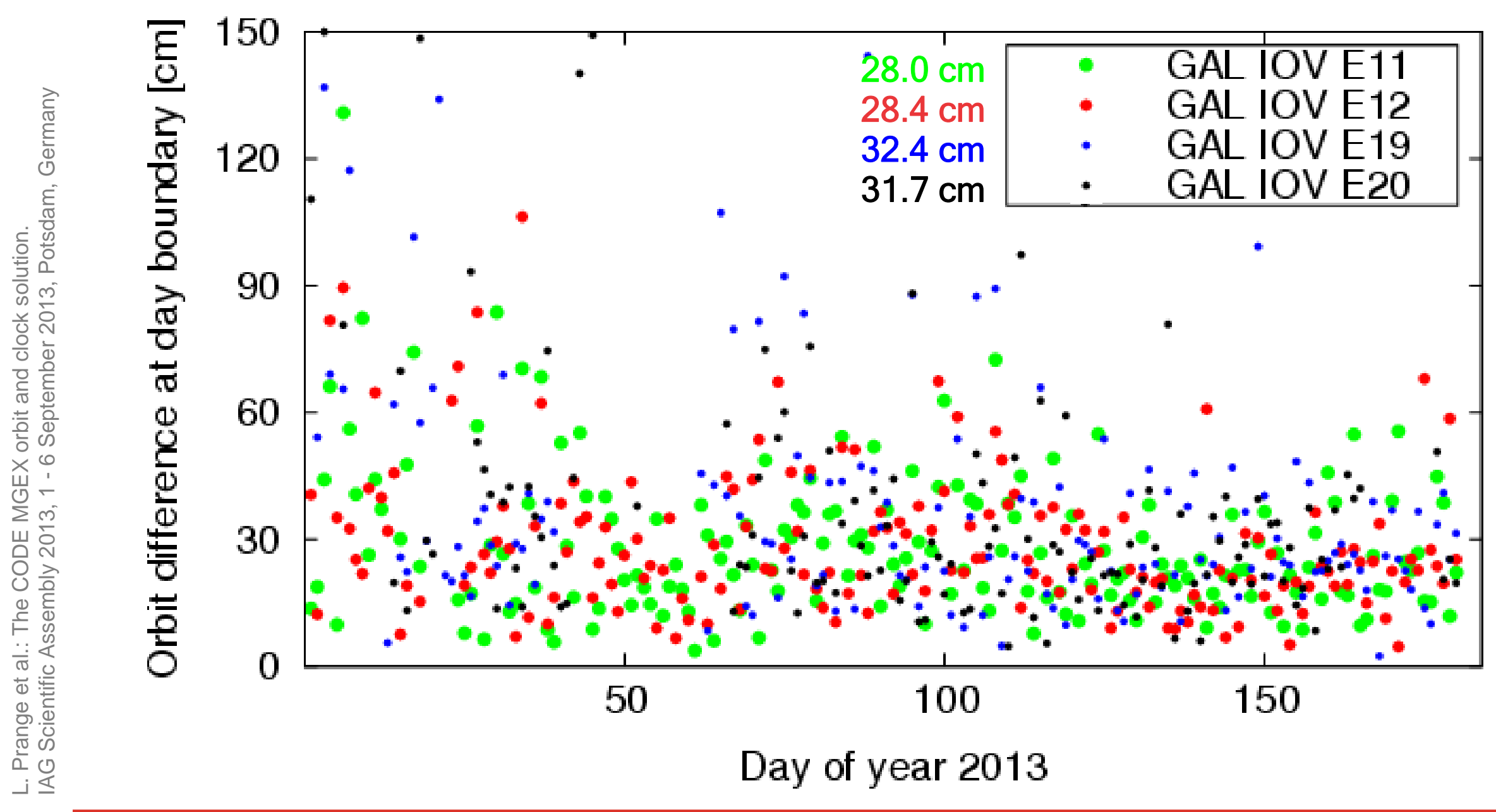

Astronomical Institute University of Bern $A / D B$ 


\section{Orbit validation: overlaps}

Arclength: 3 days

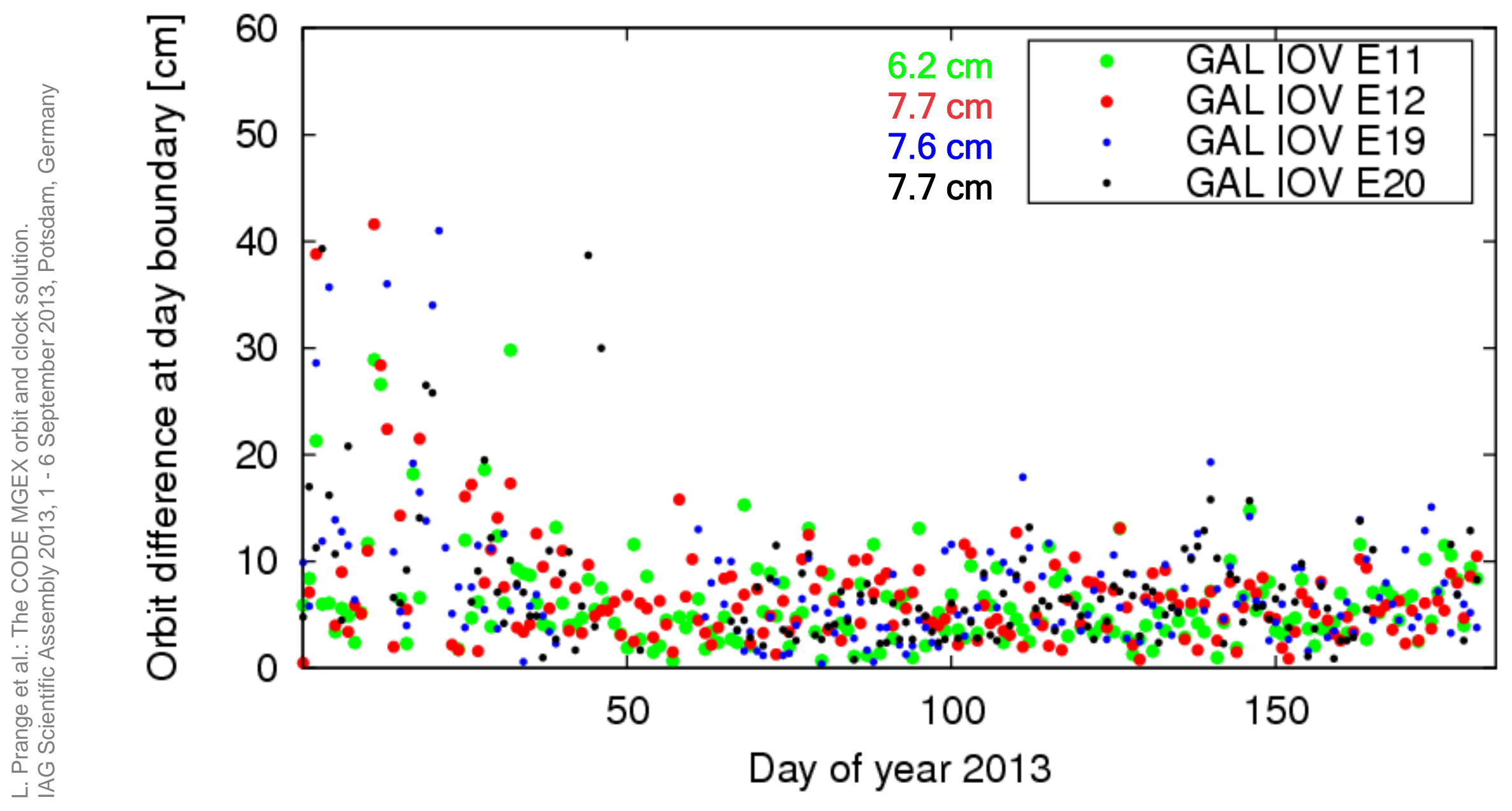

Astronomical Institute University of Bern $A V U B$ 


\section{Orbit validation : 3-day orbit fit}

Arclength: 1 day, satellite-wise

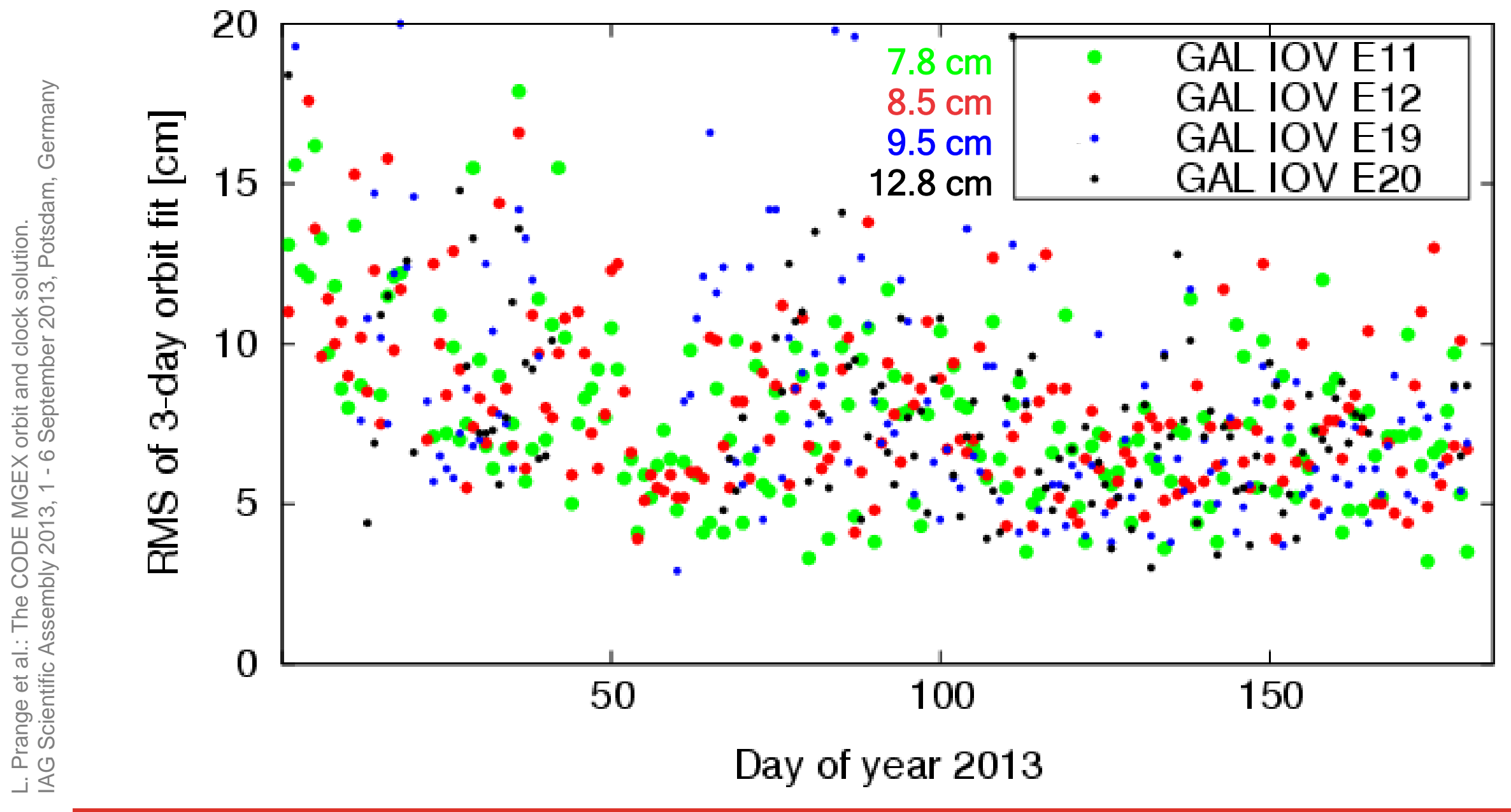

Astronomical Institute University of Bern $A / D B$ 


\section{Orbit validation : 3-day orbit fit}

Arclength: 3 days, satellite-wise

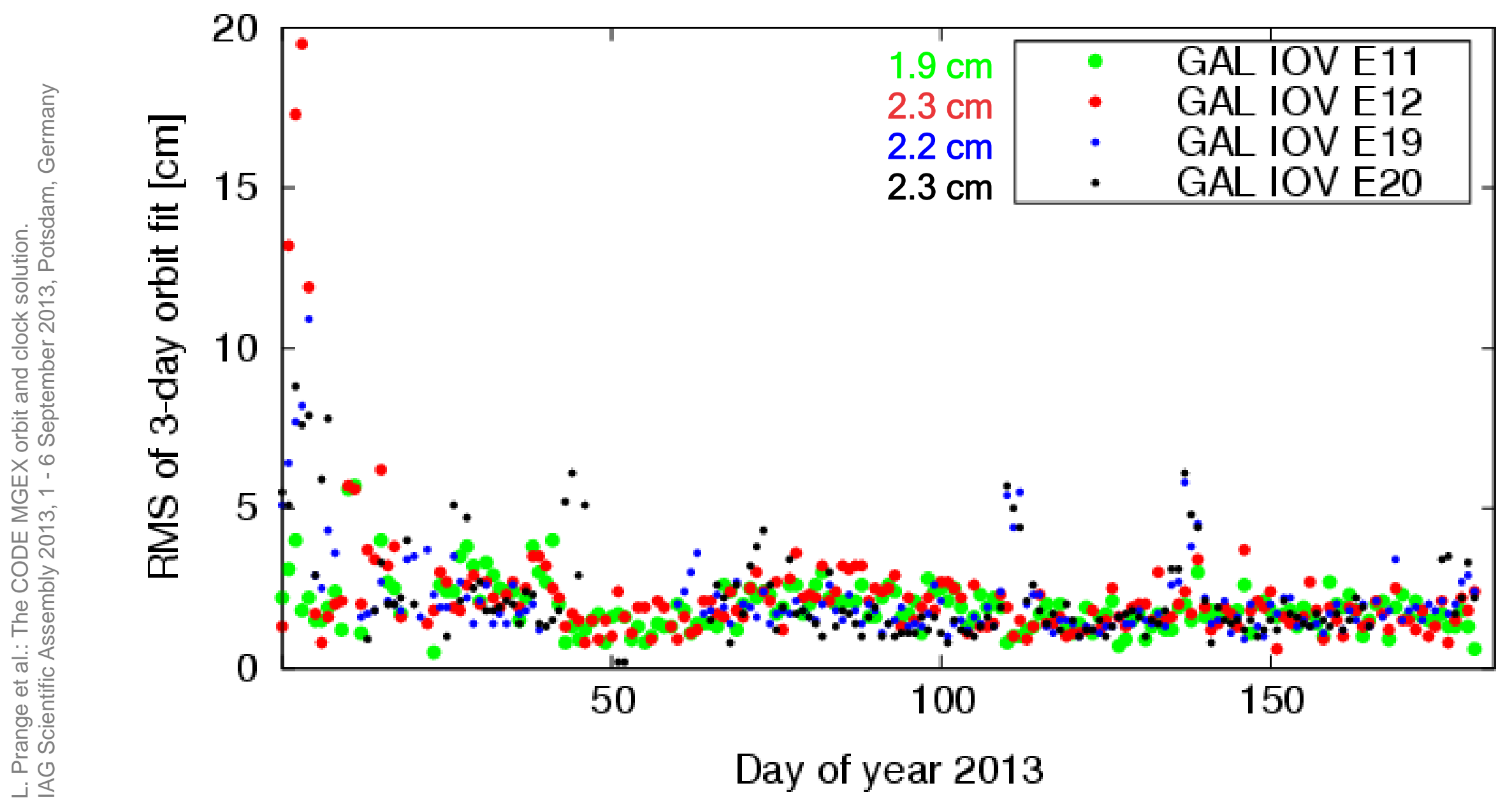

Astronomical Institute University of Bern $A / D B$ 


\section{Orbit validation : 3-day orbit fit}

Arclength: 3 days, GNSS-wise

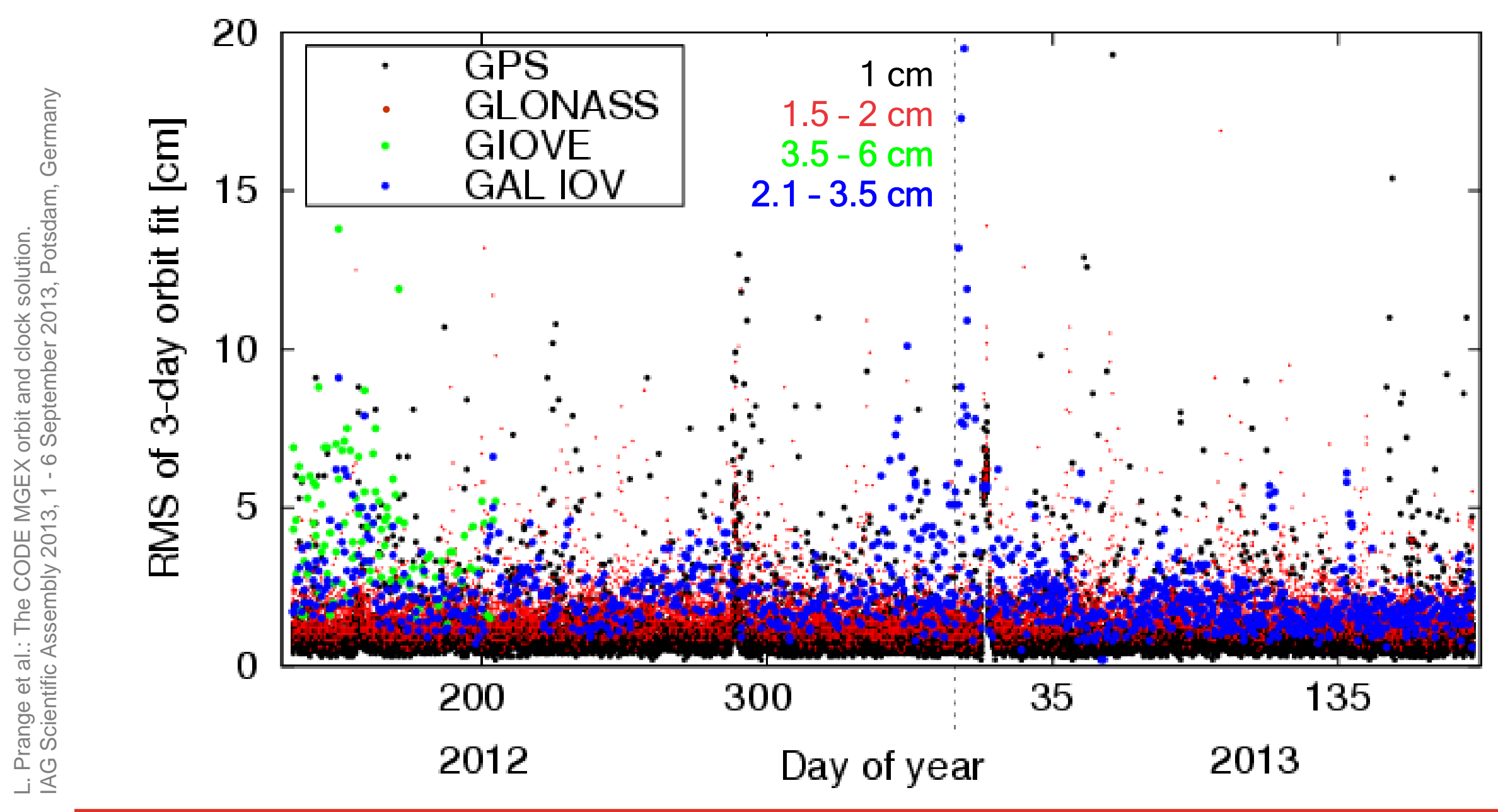




\section{Orbit validation: SLR residuals}

STD of SLR residuals per week: GNSS-wise

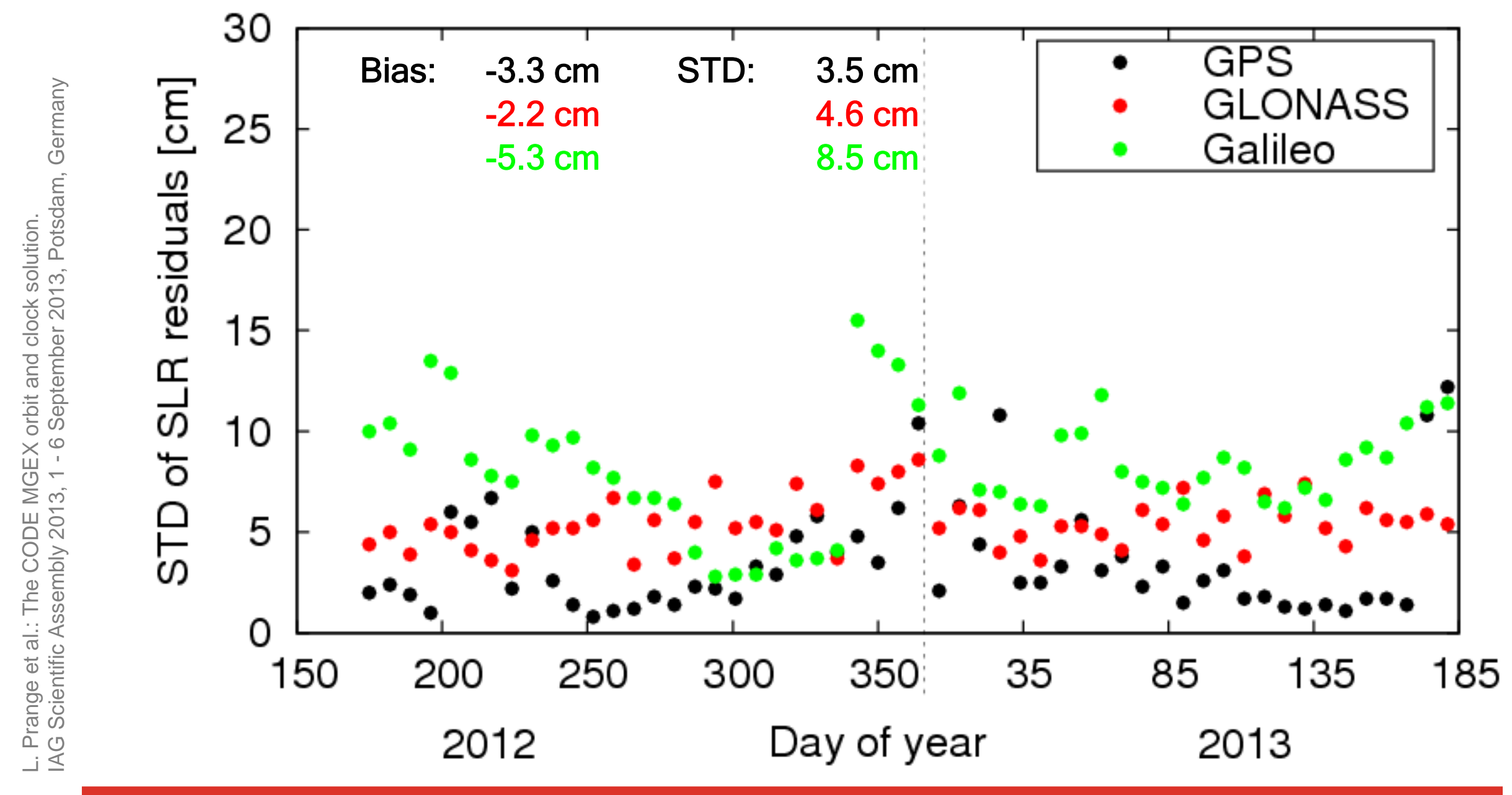




\section{Orbit validation: SLR residuals}

STD of SLR residuals per week: satellite-wise

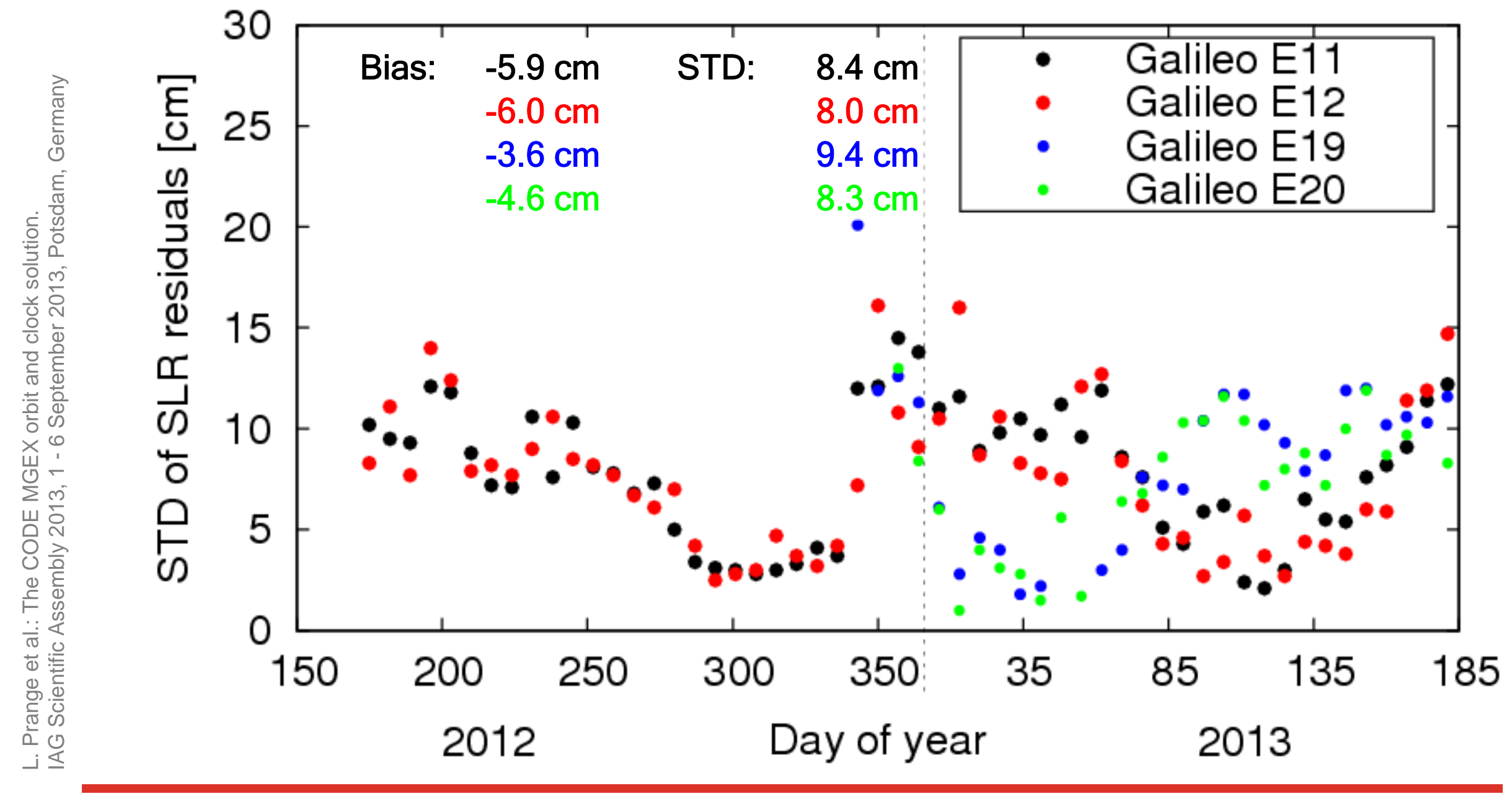




\section{Orbit validation: SLR residuals}

STD of SLR residuals per week: correlation with beta angle

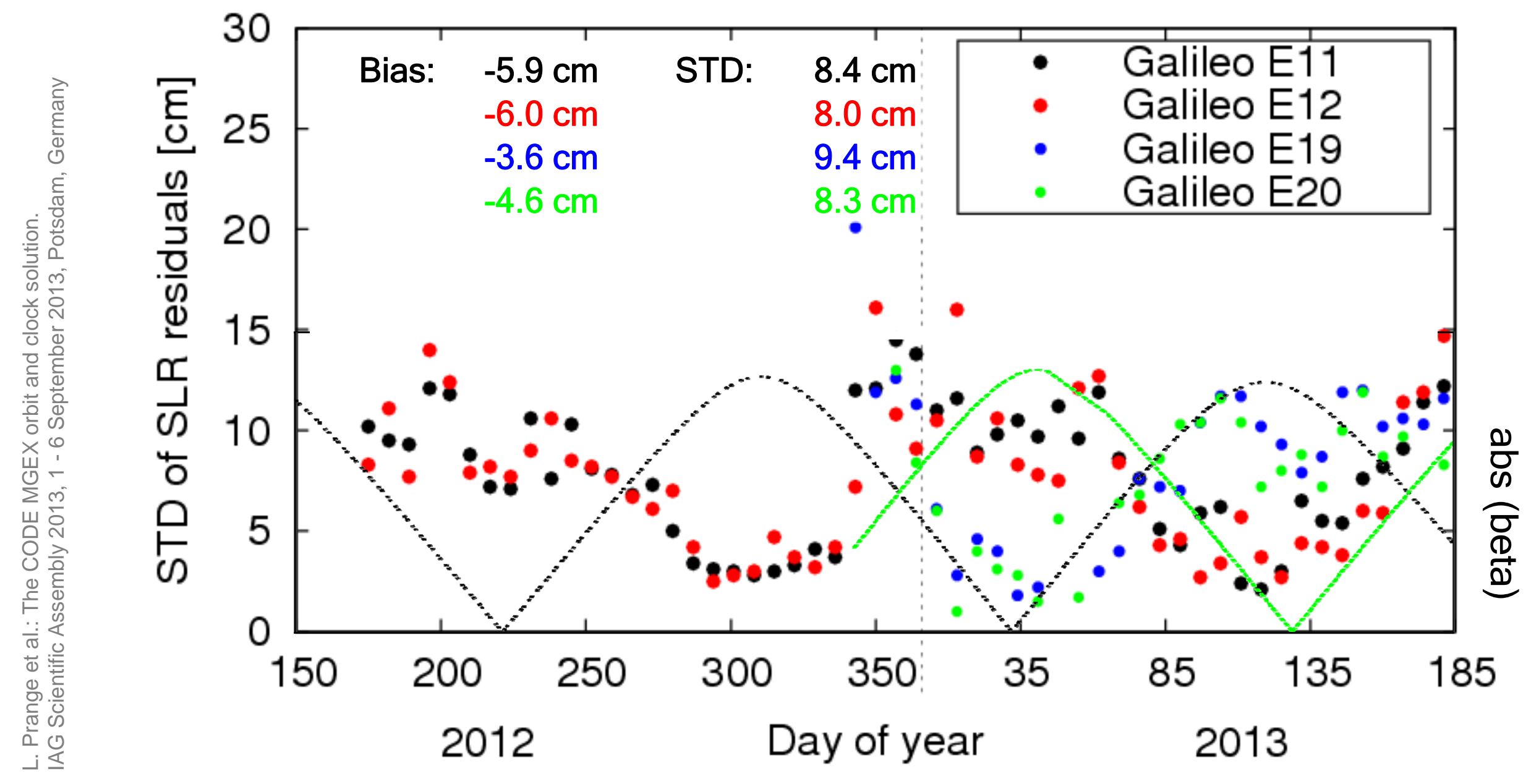




\section{CODE MGEX clock solution}

GNSS considered: $\quad$ GPS + Galileo (up to 36 satellites)

Processing mode: $\quad$ post-processing

Timespan covered: $\quad$ GPS-weeks 1710-1746 (DOY 12/288-13/180)

Number of stations: 150 (GPS), $30-40$ (Galileo)

Processing scheme: zero-difference network processing

(observable: code+phase undifferenced)

Signal frequencies: $\quad$ L1+L2 (GPS); E1(L1)+E5a (L5) (Galileo)

A priori information: orbits, ERPs, coordinates, and troposphere from

CODE MGEX orbit solution introduced as known

Reference frame: IGb08

IERS conventions: IERS2010

Product list:

epoch-wise (300s) satellite and station clock corrections in daily clock RINEX files; daily GPS-Galileo inter-system biases for mixed stations in Bernese DCB and BIAS-SINEX (BIA) format

Distribution: ftp://cddis.gsfc.nasa.gov/gnss/products/mgex/

Designator: comwwwwd.???.Z 


\section{CODE MGEX clock solution}

Clock availability: impact of station distribution

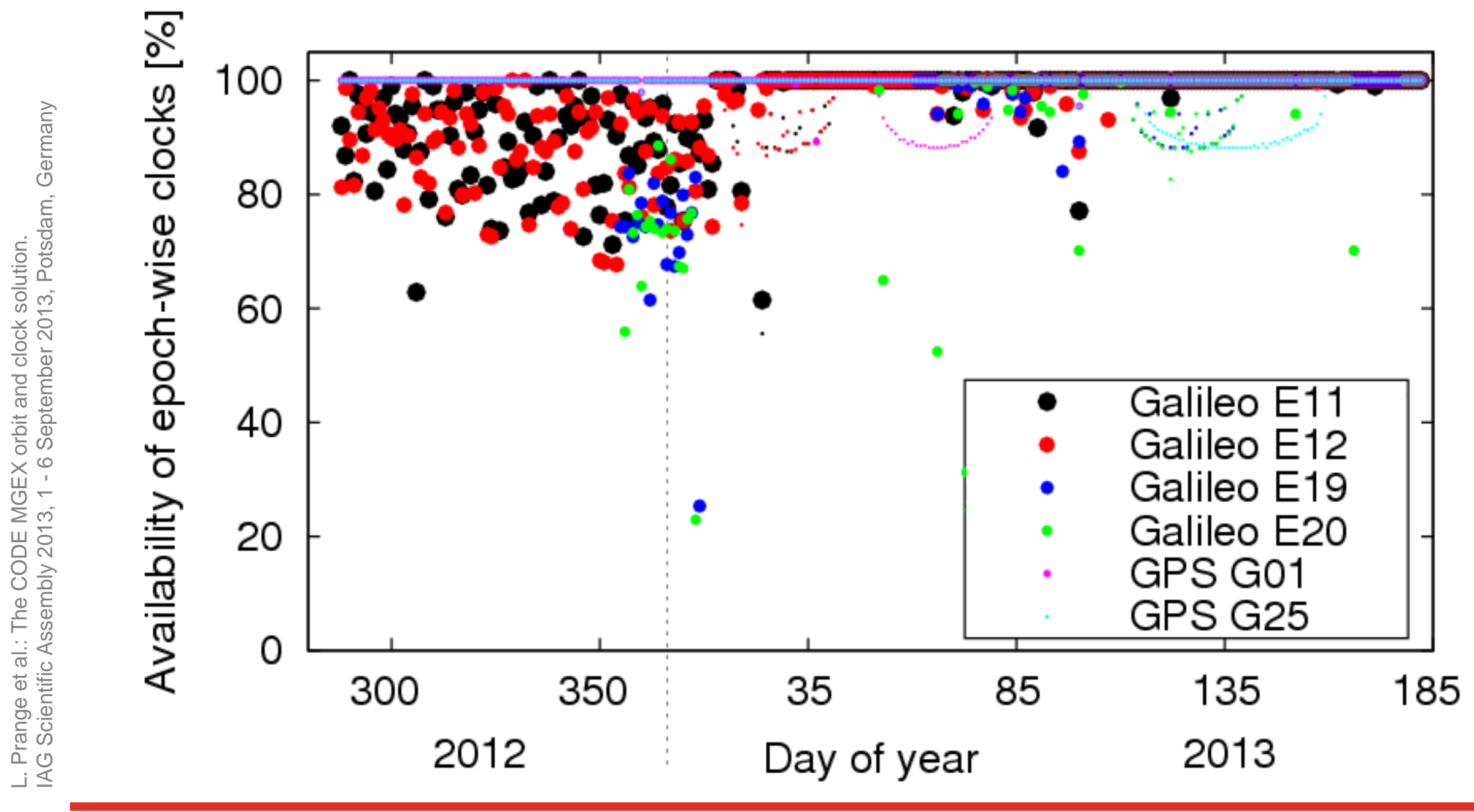




\section{CODE MGEX clock solution}

GPS-IIF: impact of sun eclipse

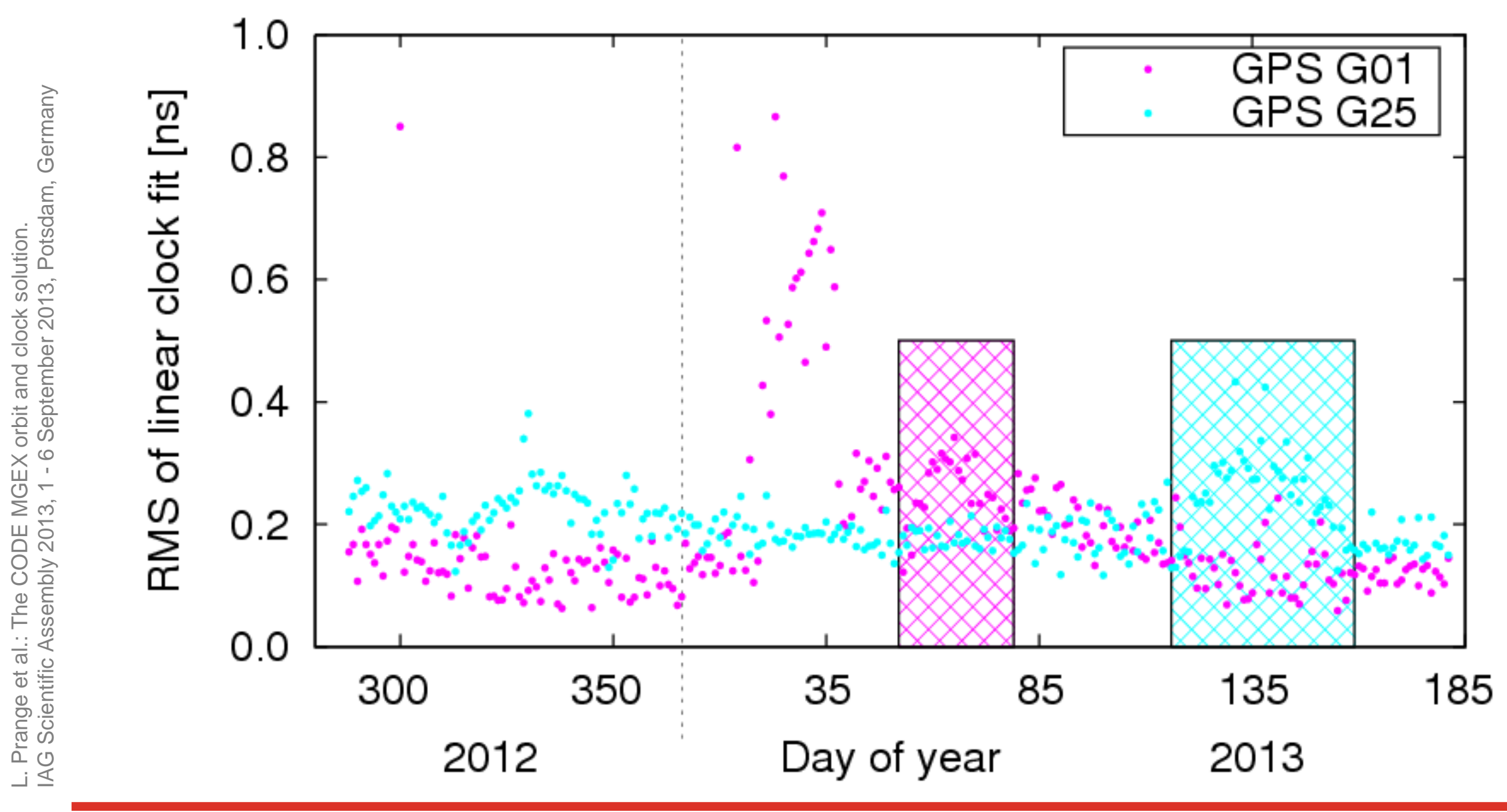




\section{CODE MGEX clock solution}

Galileo IOV: impact of sun eclipse

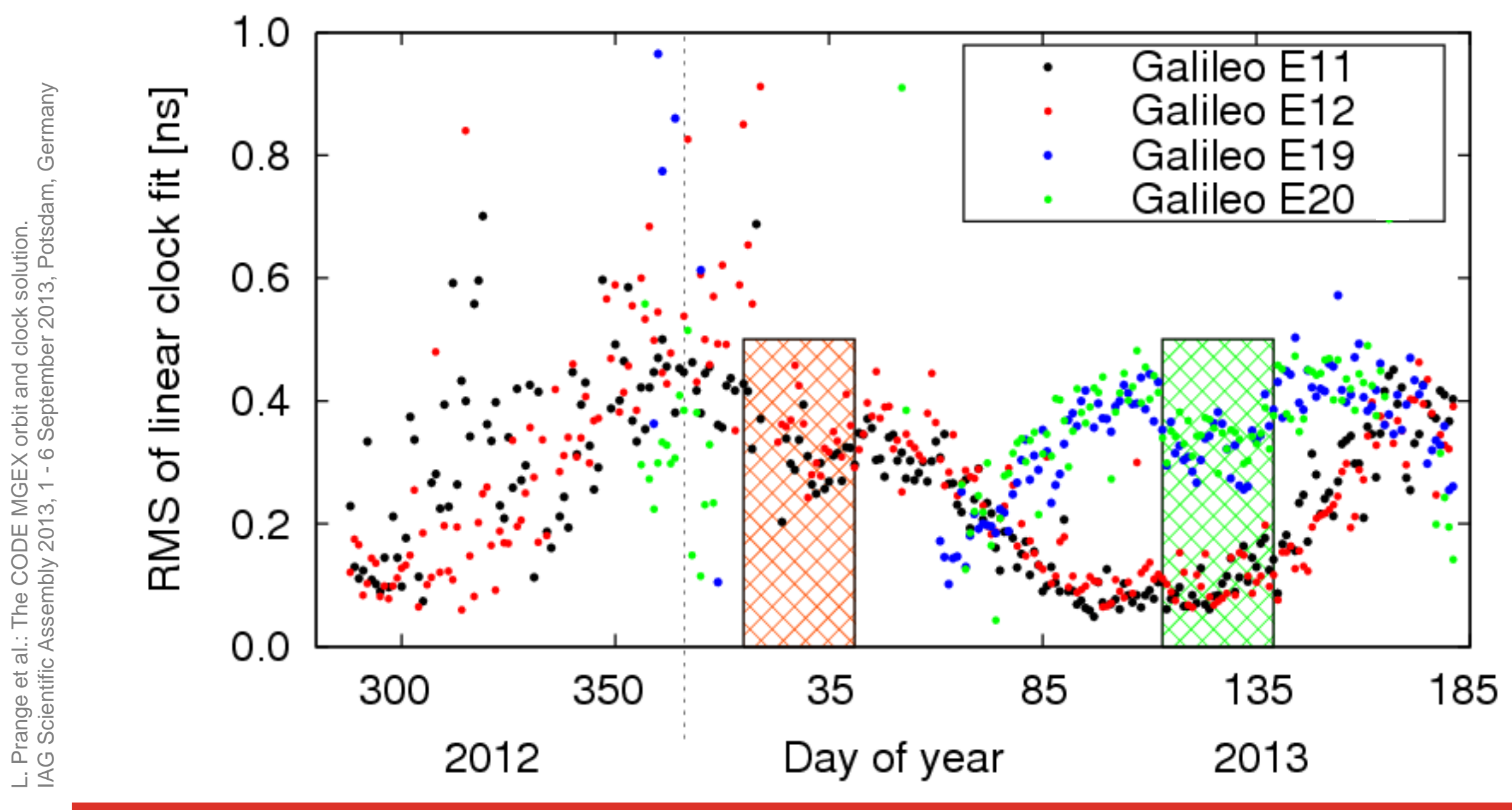




\section{CODE MGEX clock solution}

Galileo IOV: impact of sun eclipse and beta angle

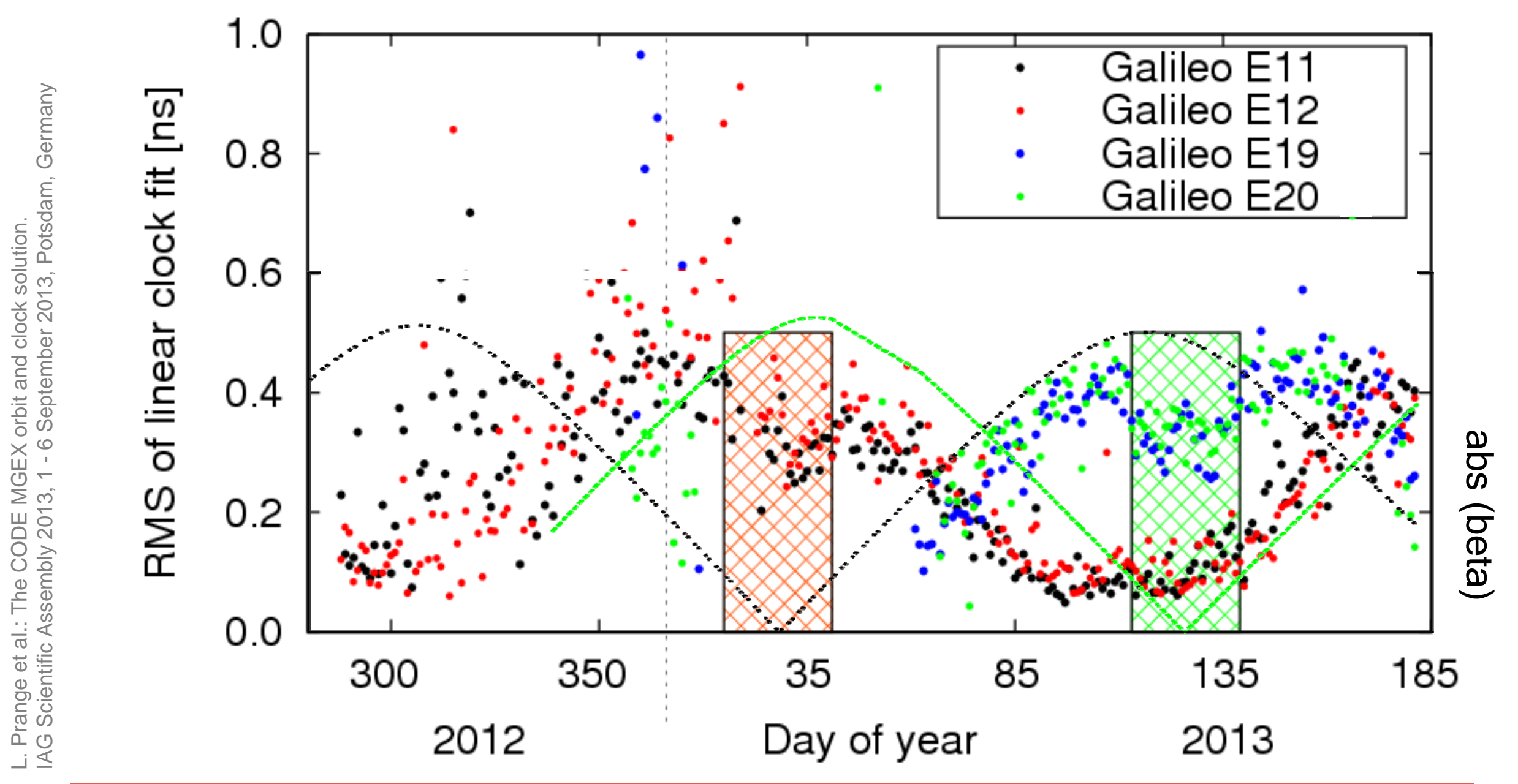




\section{CODE MGEX clock solution}

Linear fit of satellite clocks: GPS-IIF vs. Galileo IOV

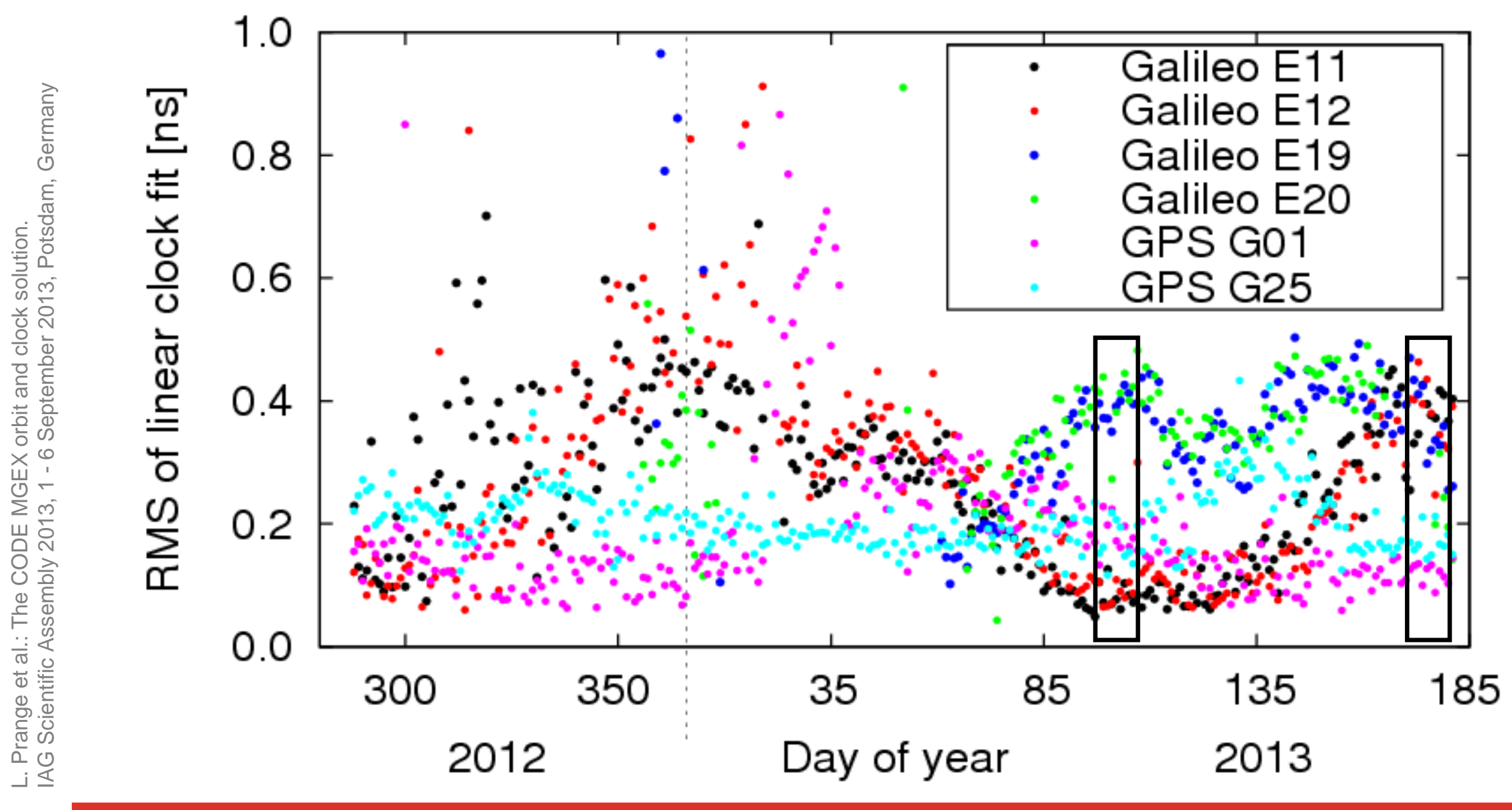




\section{CODE MGEX clock solution}

Modified Allan deviation (5 days, reference clock: BRUX)
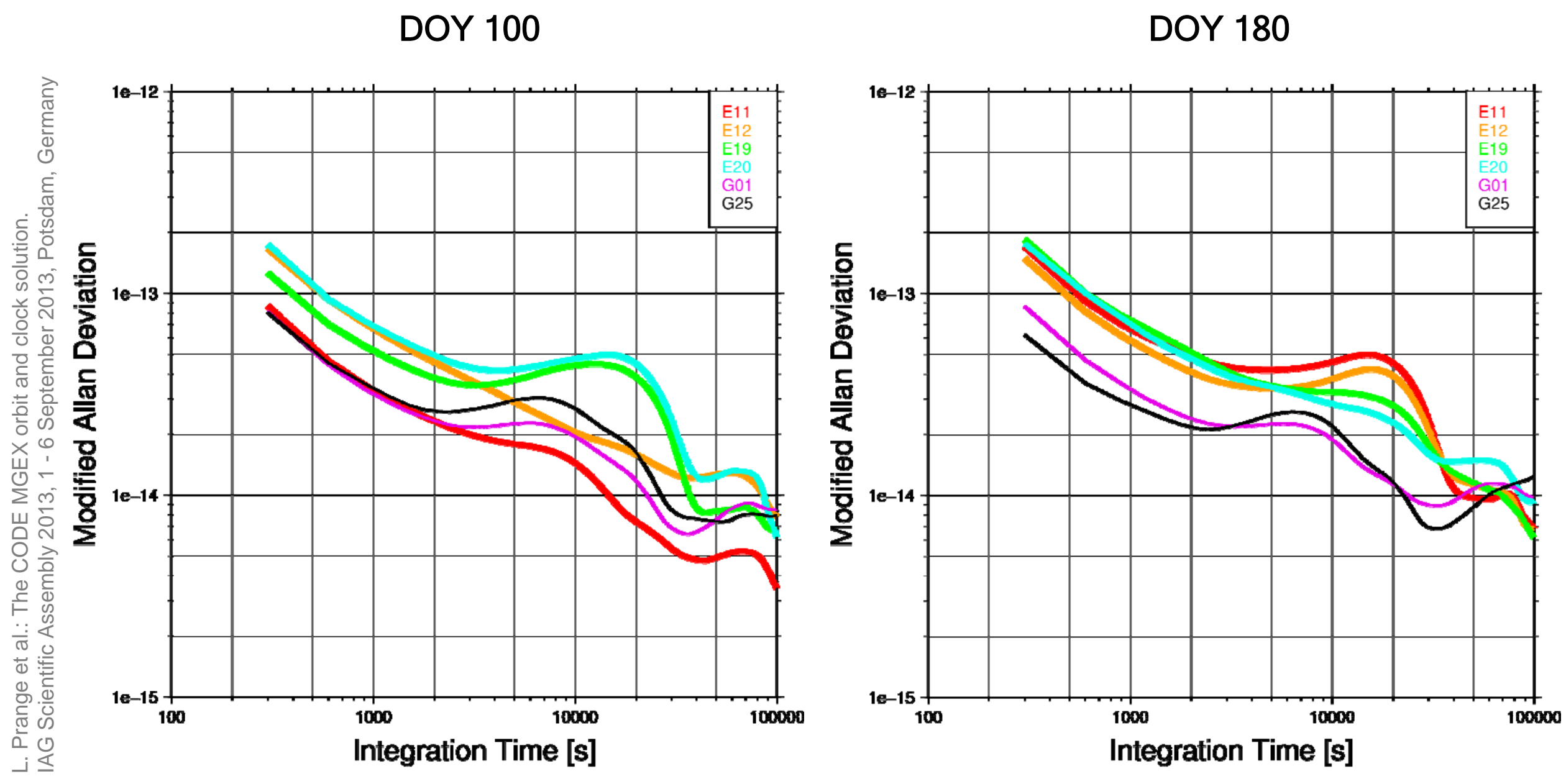

Astronomical Institute University of Bern $A / D B$ 
PPP, static mode (DOY 75 - 84)

Difference to CODE MGEX network solution (threshold $300 \mathrm{~mm}$ ):

GPS+Galileo, GPS, GPS (4 SVs), Galileo

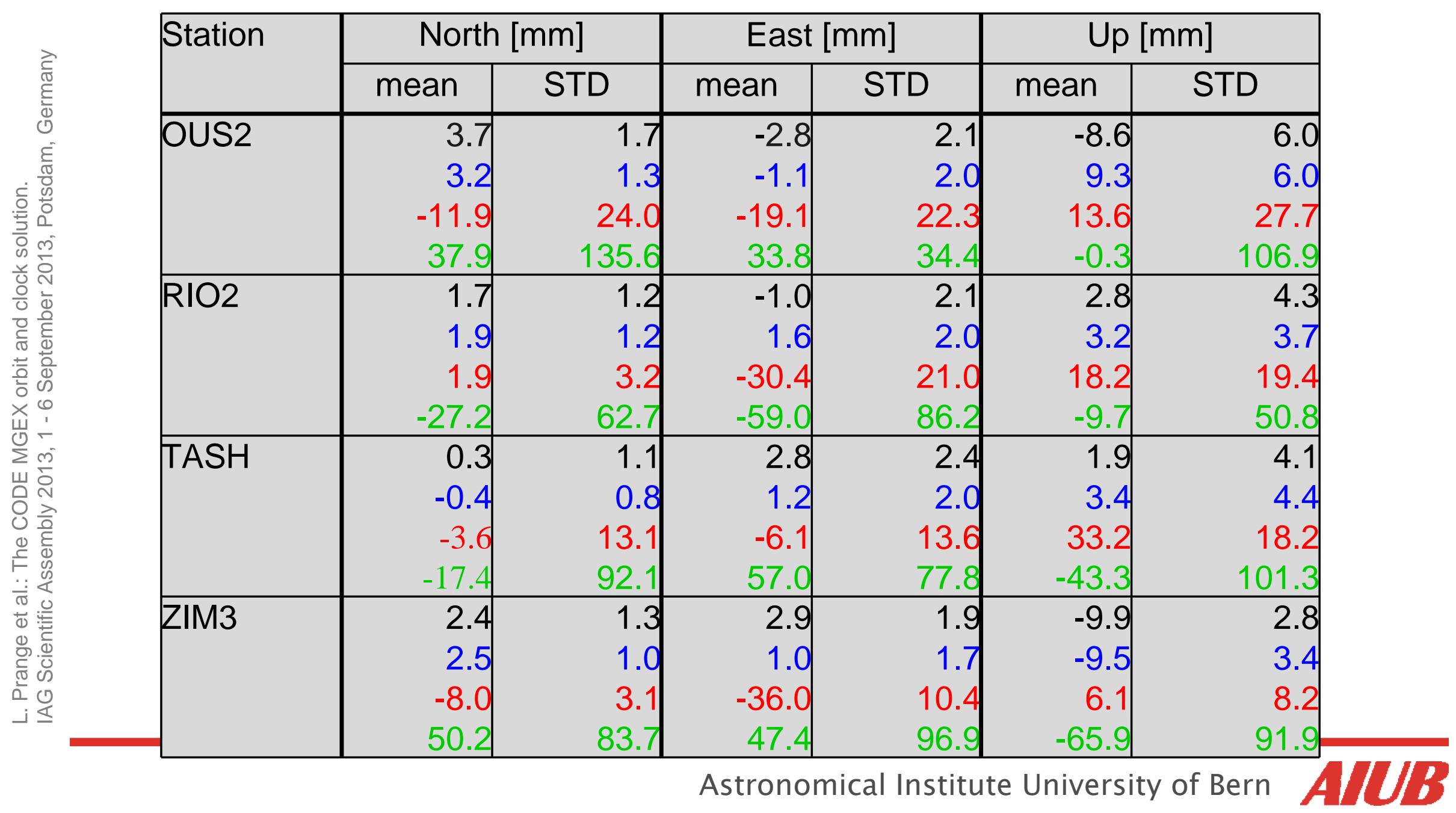




\section{PPP, static mode (DOY 75 - 84)}

Difference to CODE MGEX network solution for station ZIM3:

GPS+Galileo. GPS. GPS (4 SVs), Galileo
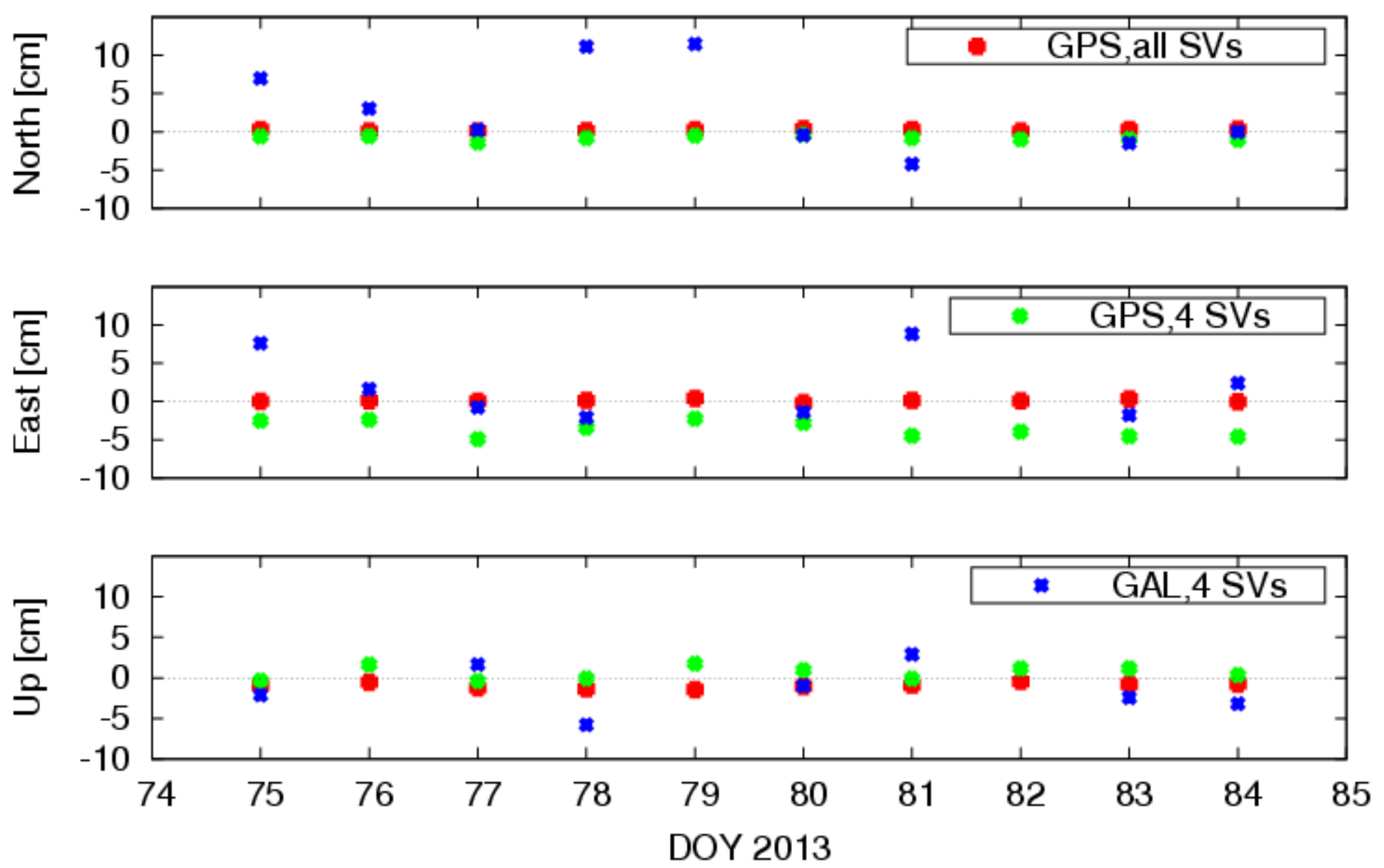
PPP, kinematic mode (DOY 75 - 84)

Difference to CODE MGEX network solution (threshold $10000 \mathrm{~mm}$ ): GPS+Galileo, GPS, GPS (4 SVs), Galileo

\begin{tabular}{|l|r|r|r|r|r|r|}
\hline Station & \multicolumn{2}{|c|}{ North $[\mathrm{mm}]$} & \multicolumn{2}{c|}{ East [mm] } & \multicolumn{2}{c|}{ Up [mm] } \\
\cline { 2 - 7 } & mean & \multicolumn{1}{c|}{ STD } & mean & \multicolumn{1}{c|}{ STD } & mean & \multicolumn{1}{c|}{ STD } \\
\hline OUS2 & 3.0 & 33.9 & -7.8 & 38.1 & 210.1 & 112.1 \\
& 2.5 & 38.6 & -7.2 & 47.7 & 216.8 & 119.9 \\
& -3132.4 & 2907.3 & -3959.6 & 3819.2 & 5066.5 & 3375.5 \\
& -104.6 & 1756.6 & 572.3 & 1036.7 & -191.3 & 2251.3 \\
\hline RIO2 & 8.0 & 36.8 & 8.2 & 39.4 & 181.5 & 141.6 \\
& 7.8 & 43.9 & 12.2 & 47.5 & 185.7 & 149.0 \\
& 357.8 & 2034.4 & 63.0 & 901.1 & 557.2 & 4917.5 \\
& -223.0 & 1283.7 & -18.9 & 516.1 & -549.8 & 1539.8 \\
\hline TASH & 1.5 & 19.4 & 0.9 & 26.6 & 112.1 & 92.8 \\
& 1.1 & 21.6 & -2.2 & 29.6 & 114.2 & 95.0 \\
& 2225.0 & 2677.2 & -2679.8 & 3040.4 & 5133.4 & 3545.8 \\
& -326.2 & 1229.5 & 270.9 & 741.1 & -7.2 & 1393.7 \\
\hline ZIM3 & 3.0 & 12.5 & 3.5 & 16.5 & 30.0 & 54.2 \\
& 3.3 & 13.2 & 1.0 & 17.0 & 30.3 & 55.1 \\
& 265.0 & 2331.1 & -360.0 & 1634.5 & -1129.9 & 4364.2 \\
& -112.2 & 690.3 & 13.8 & 369.2 & 246.7 & 944.6 \\
\hline
\end{tabular}




\section{PPP, kinematic mode (DOY 75 - 84)}

Difference to network solution: GPS, GPS (4 SVs), Galileo

Station ZIM3
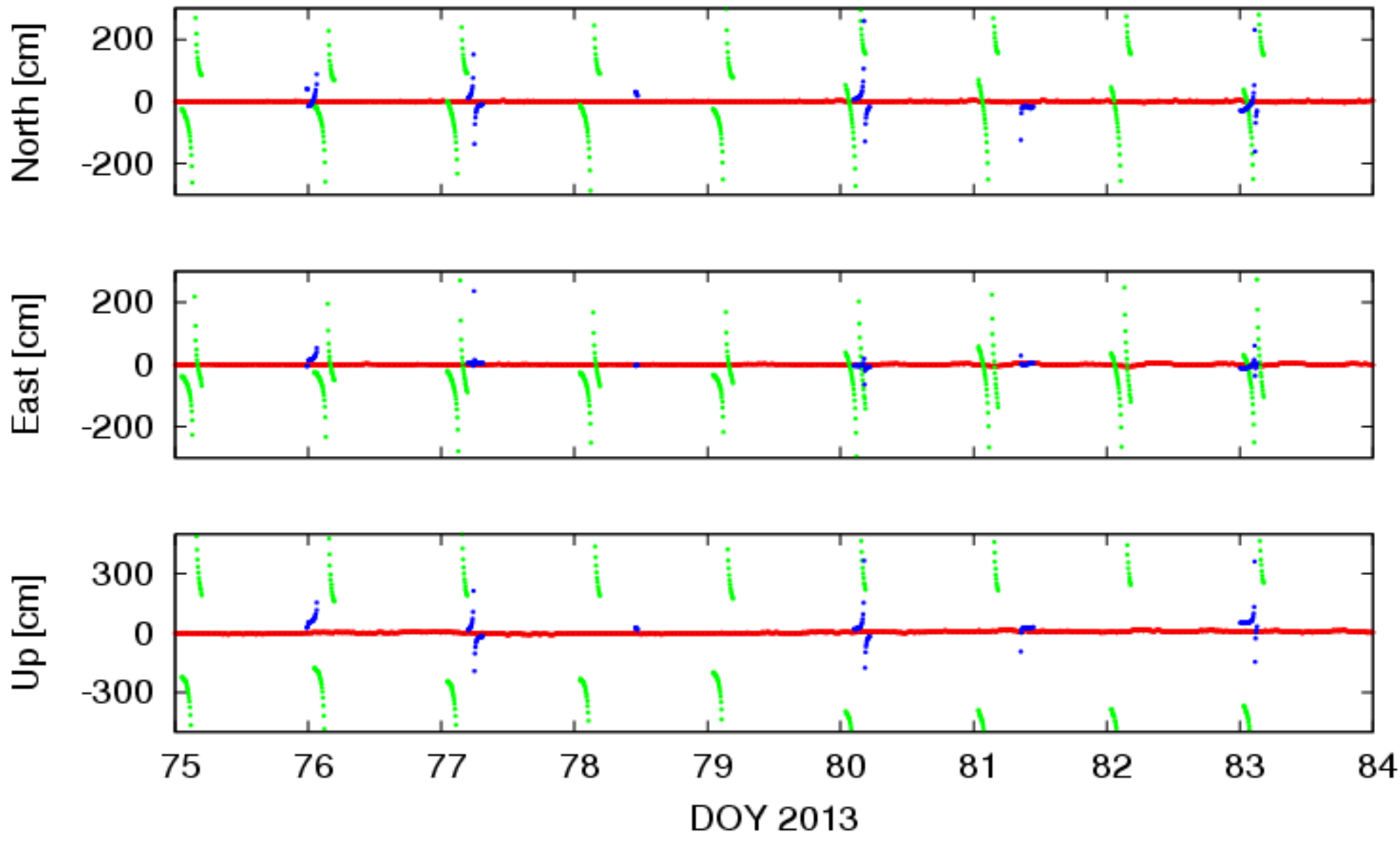


\section{PPP (DOY 75 - 84)}

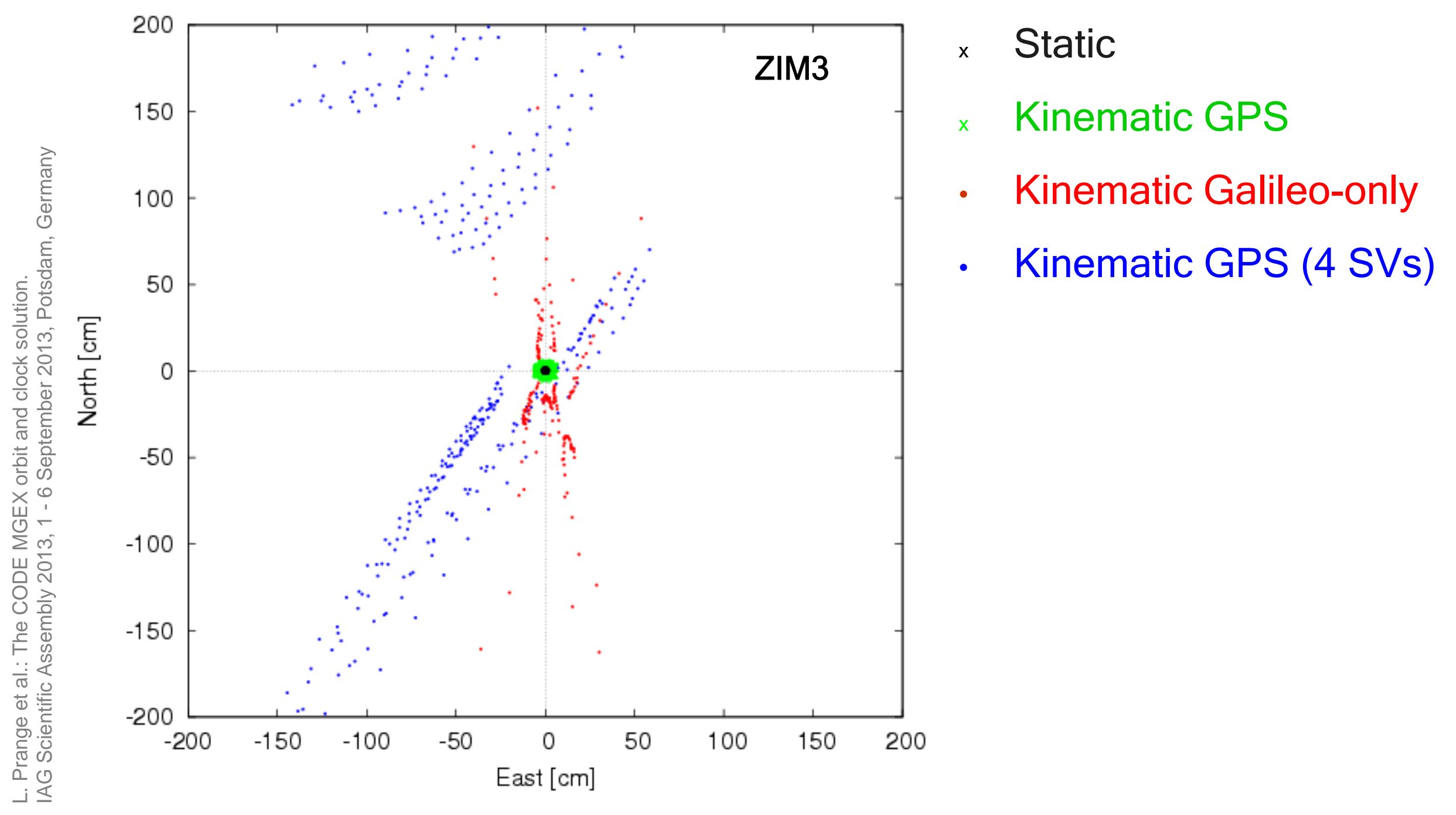




\section{Summary}

- RINEX data monitoring for IGS MGEX is well established at CODE (results are available via AIUB anonymous FTP

$=>\mathrm{ftp}: / / \mathrm{ftp}$. unibe.ch/aiub/mgex/)

- CODE provides a MGEX-based, fully integrated, triplesystem orbit solution for 2012 and 2013: GPS+GLONASS+Galileo

- Galileo orbits dramatically benefit from long arcs due to

- the inhomogeneous station distribution and

- its long orbit revolution time (>>12h)

- Future: extension to BeiDou, ... planned 


\section{Summary}

- CODE GPS+Galileo clock solutions for late 2012 and first half of 2013 are available

- Galileo-only PPP is in principle already possible

- New batches of MGEX orbit and clock solutions will be provided from time to time

- The CODE MGEX processing is done using Bernese GNSS Software 5.2

- The analysis of IGS MGEX data is very useful for understanding, integration, and exploitation of the new GNSS signals coming in RINEX3 data format. 


\title{
Thank you
}

\author{
for \\ your interest!
}

